\title{
Viscoplastic phase field simulation of microstructural evolutions under complex loadings in Ni-base superalloys
}

\author{
Anaïs Gaubert ${ }^{1, a}$, Yann Le Bouar ${ }^{2, b}$ and Alphonse Finel ${ }^{2, c}$ \\ ${ }^{1}$ Onera DMSM - 29 avenue de la Division Leclerc - 92320 Châtillon - France \\ ${ }^{2}$ Laboratoire d'Etude des Microstructures Onera/CNRS - 29 avenue de la Division Leclerc - 92320 \\ Châtillon - France \\ aanais.gaubert@onera.fr, ’yann.lebouar@onera.fr, calphonse.finel@onera.fr
}

Key words: Phase field simulations, viscoplasticity, microstructural evolution, creep, fatigue, rafting.

\begin{abstract}
An elasto-viscoplastic phase field model is proposed to study the microstructural evolutions under mechanical loadings in a Ni-base superalloy single crystal. Elastic anisotropy and inhomogeneity, as well as the description of the long range order in the $\gamma^{\prime}$ phase are included in the model. Plastic activity is introduced using a continuum crystal plasticity framework. The coupled model is used to study microstructural evolutions in superalloys under creep at high temperature. Two loading orientations are investigated, [001] and [011]. Moreover simulations under alternate fatigue loading in the [001] orientation are performed. The viscoplasticity simulations are compared to purely elastic phase field simulations and experiments in order to show the influence of plastic activity on microstructural evolutions.
\end{abstract}

\section{Introduction}

Ni-base single crystal superalloys are widely used in the aero-engine industry to manufacture turbine blades because of their outstanding mechanical properties at high temperature. We focus here on microstructural evolutions in Ni-base superalloys under mechanical loading at high temperature. The most commonly known microstructural evolution in these materials is the so-called rafting phenomenon which arises under creep loading along the orientation [001]. Phase field models are suitable to simulate microstructural evolution because they model the microstructure at mesoscale. Many studies of rafting have been proposed in the literature [1]. However, most of them do not include the plastic activity in the channels of matrix although it has been pointed out experimentally that it has a strong influence on microstructural evolution [2]. It is why phase field models have been coupled with plasticity recently $[3,4]$.

The present contribution aims at presenting such a coupled model. This model is used to simulate rafting. More complex loading are then investigated, creep along the [011] orientation and fatigue along the [001] direction. The coupled simulations are compared to elastic simulations in order to show the plastic strain influence on microstructural evolutions.

\section{The phase field model}

The phase field method consists in describing a microstructure at mesoscale by the means of continuous fields. Assuming that the superalloy can be modeled as an effective binary alloy, four fields are needed. The concentration field $c(\vec{r}, t)$ represents the local $\mathrm{Al}$ atomic concentration. Three long range order parameters fields $\eta_{i}(\vec{r}, t)$ are added to model the four variant of the $\gamma^{\prime}$ phase.

The temporal evolution of these fields is obtained solving Cahn-Hilliard and Allen-Cahn equations for the concentration field and the order parameters fields respectively:

$$
\frac{\partial c}{\partial t}(\vec{r}, t)=M \nabla^{2} \frac{\delta F}{\delta c} ; \frac{\partial \eta_{i}}{\partial t}(\vec{r}, t)=-L \frac{\delta F}{\delta \eta_{i}}
$$

where $F=F_{G L}+E_{e l}+F_{v p}$ is the total free energy of the system. 
The Ginzburg-Landau free energy. The free energy of a homogeneous microstructure $f_{\text {hom } o}\left(c,\left\{\eta_{i}\right\}\right)$ is approximated using a polynomial expansion with respect to the order parameters:

$$
f_{\text {homo }}\left(c,\left\{\eta_{i}\right\}\right)=\Delta f\left[\frac{1}{2}\left(c-c_{\gamma}\right)^{2}+\frac{B}{6}\left(c_{2}-c\right) \sum_{i} \eta_{i}^{2}-\frac{C}{3} \eta_{1} \eta_{2} \eta_{3}+\frac{D}{12} \sum_{i} \eta_{i}^{4}\right] .
$$

$\Delta f$ is the energy density scale of the model and $c_{2}$ is an arbitrary concentration chosen between the equilibrium concentrations $c_{\gamma}$ and $c_{\gamma^{\prime}}$ of the coexisting phases. $B, C, D$ constants are given by:

$$
B=2\left(c_{\gamma^{\prime}}-c_{\gamma}\right) ; C=6\left(c_{\gamma^{\prime}}-c_{\gamma}\right)\left(c_{2}-c_{\gamma}\right) ; D=6\left(c_{\gamma^{\prime}}-c_{\gamma}\right)\left(c_{\gamma^{\prime}}+2 c_{2}-3 c_{\gamma}\right) \text {. }
$$

The non equilibrium chemical free energy may be approximated by a standard Ginzburg-Landau functional:

$$
F_{G L}=\int_{V}\left[f_{\text {homo }}\left(c,\left\{\eta_{i}\right\}\right)+\frac{\lambda}{2}|\nabla c|^{2}+\frac{\beta}{2} \sum_{i}\left|\nabla \eta_{i}\right|^{2}\right] d V
$$

The elastic energy. The elastic energy of a coherent microstructure reads:

$$
E_{e l}=\frac{1}{2} \int_{V} \sigma: \varepsilon^{e l} d V
$$

where $\sigma$ is the stress tensor and $\varepsilon^{e l}$ is the elastic strain tensor. In the case of a coherent phase transformation, the elastic strain tensor is related to the total strain tensor trough $\varepsilon^{e l}(\vec{r})=\varepsilon(\vec{r})-\varepsilon^{0}(\vec{r})$ where $\varepsilon^{0}(\vec{r})$ is the stress-free strain tensor. Assuming a Vegard's law, the stress-free strain tensor can be expressed using the concentration field: $\varepsilon^{0}(\vec{r})=\varepsilon^{T} \Delta c(\vec{r})$ where $\Delta c(\vec{r})=c(\vec{r})-\bar{c}, \bar{c}$ being the average concentration. The transformation strain tensor $\varepsilon^{T}$ is diagonal and its components are related to the misfit $\delta$.

The strain tensor is computed by solving the mechanical equilibrium equations:

$\operatorname{div} \sigma=0$ and $\bar{\sigma}=\sigma^{a}$ in the case of an aplied stress.

An iteratif scheme is needed to solve mechanical equilibrium. It is detailled in [4] .The strain tensor is related to the stress tensor by Hooke's law: $\sigma=\lambda: \varepsilon^{e l}$. In the present model, the inhomogeneity in elastic constants is taken into account. This is obtained by making the tensor $\lambda$ dependent on the local value of the field:

$$
\lambda(c(\vec{r}))=\bar{\lambda}+\lambda^{\prime} \Delta c(\vec{r}),
$$

the $\bar{\lambda}$ and $\lambda^{\prime}$ tensors being related to the elastic constants of both phases and their volume fraction.

Numerical inputs. The phase field model requires four types of numerical inputs: numerical inputs for the homogeneous free energy density, for elasticity, for interface energy and diffusion coefficients. The identification of these quantities is detailed in [4].

\section{Phenomenological viscoplasticity}

The total strain tensor $\varepsilon$ can be decomposed into an elastic and a plastic part: $\varepsilon=\varepsilon^{e l}+\varepsilon^{p}$. Single crystal superalloys have an anisotropic viscoplastic behavior at high temperature. The crystal plasticity framework is therefore used [5]. In this model, the plastic deformation tensor reads as the sum of the strain over all slip systems:

$$
\varepsilon^{p}=\sum_{s} \gamma^{s} m^{s}
$$


where $m^{s}$ repersents the orientation tensor for the slip system s. The plastic shear on each slip system is determined from a constitutive model based on the Schmid criterion. A Norton's flow rule is used:

$$
\gamma^{s}=\left\langle\frac{\left|\tau^{s}-x^{s}\right|-r_{0}^{s}}{k}\right\rangle^{n} \operatorname{sign}\left(\tau^{s}-x^{s}\right) .
$$

In this equation, $\tau^{s}$ is the resolved shear stress on the slip system $\mathrm{s}$ and $x^{s}$ denotes the kinematic hardening, isotropic hardening being negliged in the case of superalloys [5]. A non-linear evolution law is adopted for the kinematic hardening:

$$
x^{s}=c^{s} \alpha^{s} \text { with } \dot{\alpha}^{s}=\dot{\gamma}^{s}-d^{s}\left|\dot{\gamma}^{s}\right| \alpha^{s}
$$

where $\alpha^{s}$ denotes the kinematic hardening state variable and $c^{s}$ and $d^{s}$ are two material parameters.

Single crystal superalloys deform via slip in the octahedral planes due to their crystallography. However, for $<111>$ oriented specimens, the macroscopic slip occur via cubic systems although they are contested at the microscale [6]. In the model, cubic slip systems are taken into account.

\section{Coupling between the phase field model and viscoplasticity}

When tacking plasticity into account, the elastic energy reads:

$$
E_{e l}=\frac{1}{2} \int_{V} \lambda:\left(\varepsilon-\varepsilon^{0}-\varepsilon^{p}\right):\left(\varepsilon-\varepsilon^{0}-\varepsilon^{p}\right) d V .
$$

Moreover, an hardening free energy density appears in the model:

$$
f_{v p}=\frac{1}{2} \sum_{s} c^{s}\left(\alpha^{s}\right)^{2}
$$

The driving forces have to be derived from these energies in order to compute the evolution of the fields $c(\vec{r})$ and $\eta_{i}(\vec{r})$.

Viscoplasticity parameters in the phase field model. The two phases $\gamma$ and $\gamma^{\prime}$ have a different mechanical behavior. The plastic activity mainly occurs in the matrix $\gamma$ channels while the $\gamma^{\prime}$ precipitates remain elastic under creep loading. It is why different material parameters have to be chosen for the two phases. Within the elasto-viscoplastic framework, the simplest way to ensure an elastic behavior for the $\gamma^{\prime}$ phase is to set the initial threshold $r_{0 \gamma^{\prime}}$ to a very high value for all slip systems. The threshold is thus interpolated in the $\gamma / \gamma^{\prime}$ interface:

$$
r_{0}(\vec{r})=\bar{r}_{0}+r_{0}{ }^{\prime} \tanh \left(\theta \frac{c(\vec{r})-\bar{c}}{c_{\gamma^{\prime}}-c_{\gamma}}\right)
$$

with $\bar{r}_{0}=\frac{r_{0 \gamma}+r_{0 \gamma^{\prime}}}{2}$ and $r_{0}{ }^{\prime}=\frac{r_{0_{\gamma}}-r_{0 \gamma^{\prime}}}{2}$. The other viscoplastic material parameters are taken constant. This choice implies that the hardening driving force $\frac{\delta F_{v p}}{\delta c}$ vanishes. Consequently, in the model, the coupling between plastic activity and microstructure arises through the elastic driving force.

The material parameters for both phases have been identified on monotonic and cyclic experiments on the AM1 superalloy. The identification procedure is explained in [4]. 


\section{Simulation of microstructural evolution under creep along the [001] orientation}

The first simulations concerns microstructural evolutions under creep at $1050^{\circ} \mathrm{C}, 150 \mathrm{MPa}$ along the [001] orientation. A rafted microstructure is expected. The simulations are performed in $3 \mathrm{D}$ on a $64 \times 64 \times 64$ points mesh. Figure 1 shows the results of the elastic simulation and figure 2 presents the results of the elasto-viscoplastic one. The tensile axis is horizontal. In both cases, the microstructure evolves in order to form platelets perpendicular to the tensile axis. However, two differences can be noted between the two simulations. Firstly, the rafting kinetic is faster in the elasto-viscoplastic simulation. Indeed, rafting is completed after $1.75 \mathrm{~h}$ in elasticity whereas only $0.61 \mathrm{~h}$ are needed with plasticity. Secondly, plasticity has an influence on the precipitates shape. The rafts obtained in elasticity are straighter than the rafts obtained in elasto-viscoplasticity which leads to more realistic shapes. Figure 3 presents the plastic strain field. After $0.44 \mathrm{~h}$, the plastic activity is concentrated in the vertical channels of matrix. An intermediate value of plastic deformation is observed near the corners of the precipitates. The plastic activity in these areas explains the curved shape of the precipitates.
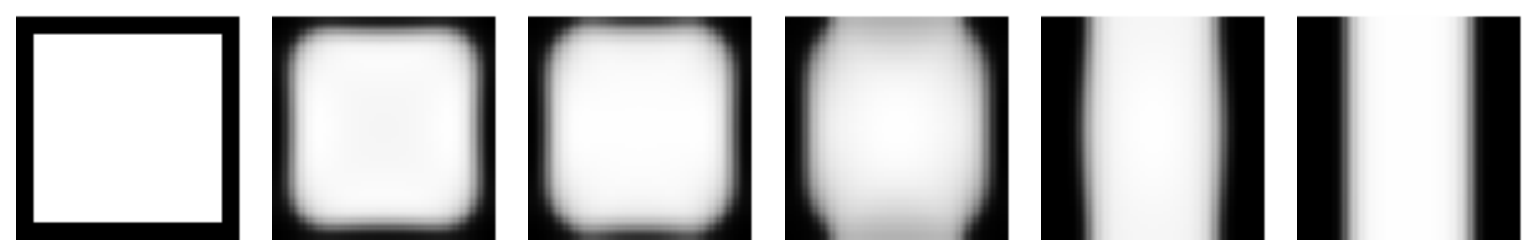

Figure 1: Microstructural evolution simulated with the elastic phase field model under creep along the [001] orientation at $0 \mathrm{~h}, 0.89 \mathrm{~h}, 1.31 \mathrm{~h}, 1.75 \mathrm{~h}, 3.50 \mathrm{~h}$, and $8.90 \mathrm{~h}$ respectively. Pictures represent the central section of the simulation box. The simulation size is $526 \mathrm{~nm} \times 526 \mathrm{nmx} 526 \mathrm{~nm}$.

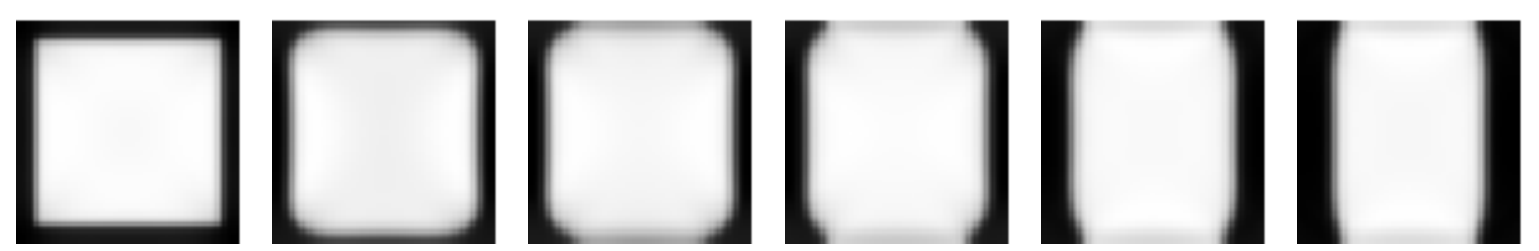

Figure 2: Microstructural evolution simulated with the elasto-viscoplastic phase field model under creep along the [001] orientation at $0 \mathrm{~h}, 0.44 \mathrm{~h}, 0.61 \mathrm{~h}, 0.89 \mathrm{~h}, 1.75 \mathrm{~h}, 8.90 \mathrm{~h}$ respectively. Pictures represent the central section of the simulation box. The simulation size is $526 \mathrm{~nm} \times 526 \mathrm{~nm} \times 526 \mathrm{~nm}$.
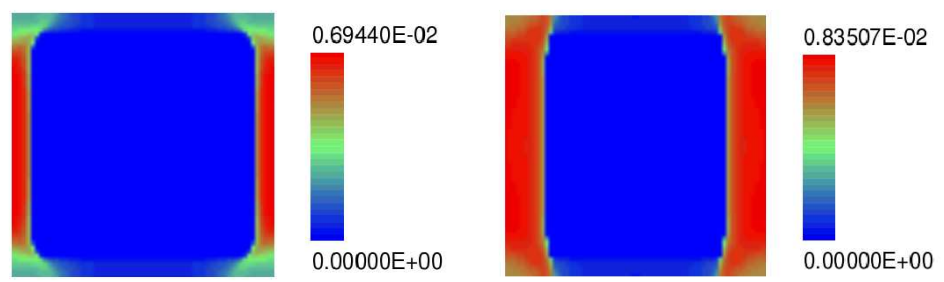

Figure 3: Plastic deformation field $\varepsilon_{11}^{p}$ at $0.44 \mathrm{~h}$ and $8.90 \mathrm{~h}$ respectively.

\section{Simulation of microstructural evolution under creep along the [011] orientation}

This section presents simulation results for creep at $1050^{\circ} \mathrm{C}, 150 \mathrm{MPa}$ along the [011] orientation. The elastic results, figure 4 , show that the microstructure coarsens along the $\mathrm{z}$ axis, the precipitates section remaining cubic. The elasto-viscoplastic simulation, figure 5, exhibits a different result. A coarsening along the $\mathrm{z}$ axis is still observed but the section of the precipitates is different. At the beginning (after $0.89 \mathrm{~h}$ ), it is cubic but it evolves and in the latest stages the [110] orientation becomes energetically equivalent to the [100] and [010] directions.

The elasto-viscoplastic result is coherent with experimental observations [7]. This author has pointed out the coexistence of three platelets families: platelets normal to the directions $<100>$, $<010>$ and $<110>$. These orientations correspond to the orientations given by the elasto-viscoplastic simulation. 

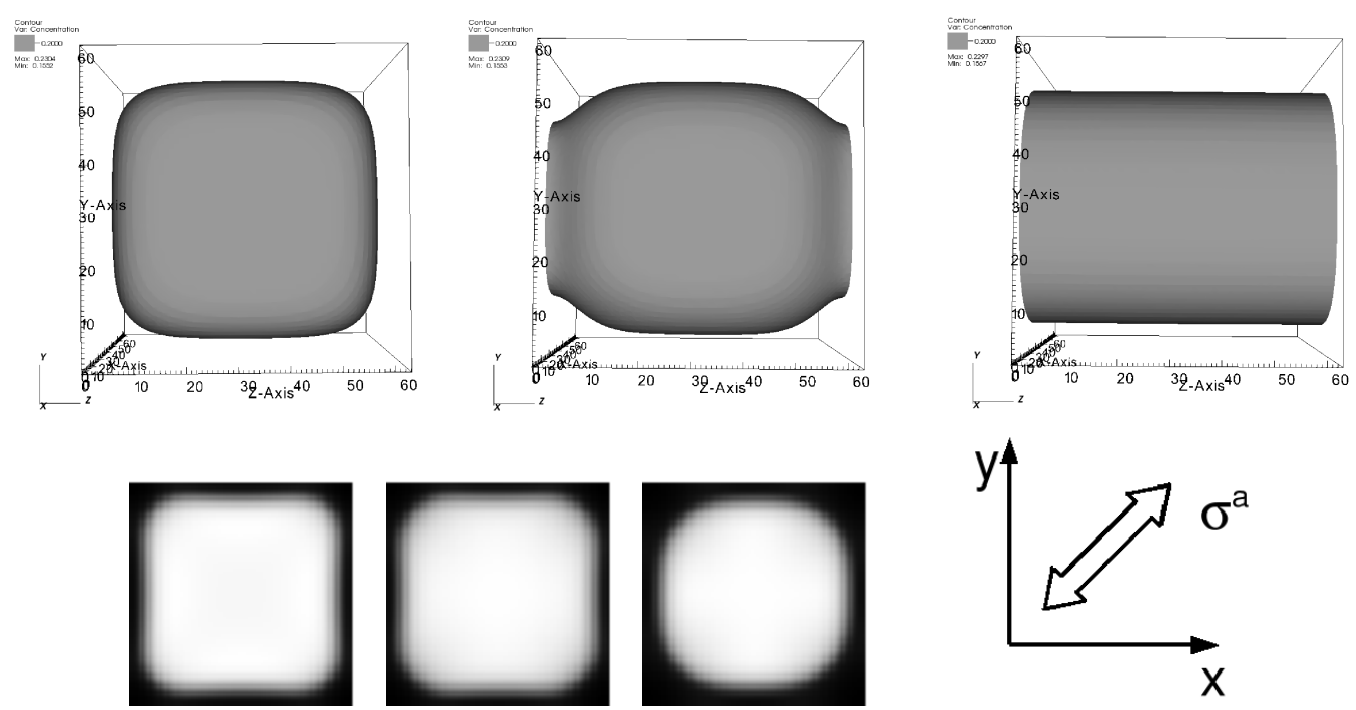

Figure 4: Microstructural evolution simulated with the elastic phase field model under creep along the [011] orientation at $0.89 \mathrm{~h}, 1.75 \mathrm{~h}$ and $8.90 \mathrm{~h}$. Pictures of the lower line represent the central section of the simulating box. The simulation size is $526 \mathrm{~nm} \times 526 \mathrm{~nm} \times 526 \mathrm{~nm}$.

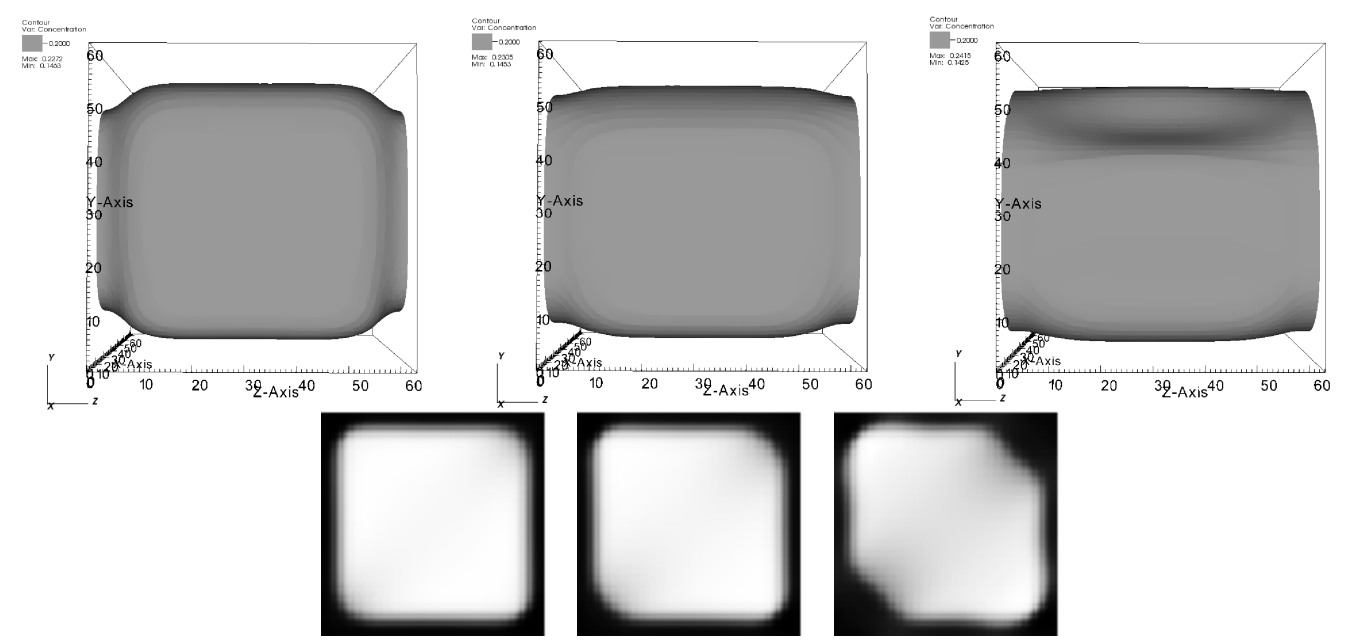

Figure 5: Microstructural evolution simulated with the elasto-viscoplastic phase field model under creep along the [011] orientation at $0.89 \mathrm{~h}, 1.75 \mathrm{~h}$ and $8.9 \mathrm{~h}$. Pictures of the lower line represent the central section of the simulation box. The simulation size is $526 \mathrm{nmx} 526 \mathrm{~nm} \times 526 \mathrm{~nm}$.

\section{Simulation of microstructural evolution under alternate fatigue in the [001] orientation}

The influence of an alternate fatigue loading at $950^{\circ} \mathrm{C}$ has been simulated. The applied deformation is $0.9 \%$, the deformation rate is $10^{-5} \mathrm{~s}^{-1}$. Simulations are performed in $2 \mathrm{D}$ on a regular mesh containing $512 \times 512$ points. The initial configuration is generated from a disordered state with stress-free boundary conditions with the elastic phase field model. Annealing at $1200^{\circ} \mathrm{C}$ during 15 minutes followed by annealing at $950^{\circ} \mathrm{C}$ during $1.5 \mathrm{~h}$ is simulated to obtain the cuboidale microstructure.

Figure 6 presents the results of the elastic and elasto-viscoplastic simulations after 15 cycles. With the elastic model the precipitates coarsen but the directions $<100>$ and $<010>$ are still the preferential orientations of the microstructure. In the elasto-viscoplastic case, a microstructure disorientation is observed. The microstructure tends to be aligned to directions at $45^{\circ}$ from the cubic axis. The plastic deformation field after 15 cycles is plotted on figure 7 . The plastic strain is oriented at $45^{\circ}$ from the cubic directions. Consequently, the plastic strain field explains the orientation taken by the microstructure. 
This result is in agreement with the microstructure evolution observed experimentally [8]. When the loading rate is slow enough, a $45^{\circ}$ oriented microstructure is observed as in the elastoviscoplastic phase field simulation. This results shows that it is necessary to take the plastic activity influence into account to reproduce the experimental microstructure evolution.
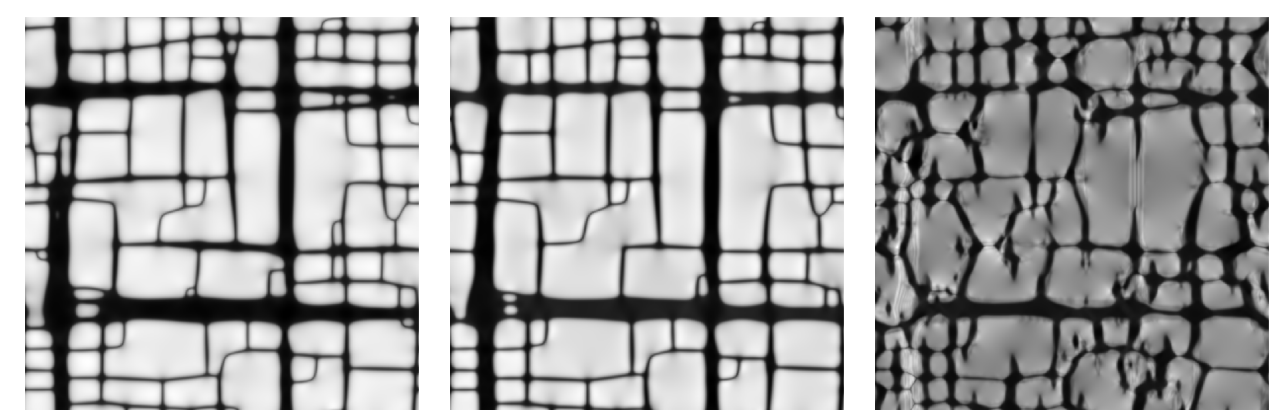

Figure 6: Microstructural evolution under alternate fatigue along the [001] direction. The left picture is the initial configuration. The middle picture represents the evolution after 15 cycles simulated with the elastic model and the right picture the simulation with the elasto-viscoplastic model.
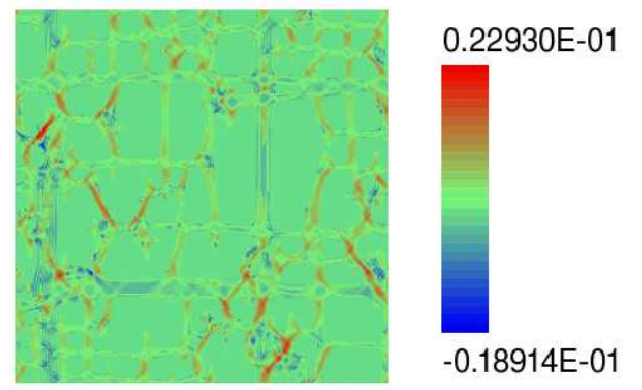

Figure 7: Plastic deformation field $\varepsilon_{11}^{p}$ after 15 cycles.

\section{Conclusion}

We have developed a phase field model coupled to a continuum crystal plasticity model. This model has been applied to study microstructural evolutions in Ni-base single crystal superalloys. Various loading conditions have been simulated: creep along the [001] orientation, creep along the [011] orientation and alternate fatigue along the [001] orientation. For all cases, elastic and elastoviscoplastic simulations have been compared to experiments. The simulations have shown that it is necessary to take plasticity into account to get the proper microstructural evolution.

\section{References}

[1] D.Y. Li, L.Q. Chen: Scripta Mat. Vol. 37 (1997), p. 1271

[2] M. Fahrmann, E. Fahrmann, O. Paris, P. Fratzl, T.M. Pollock: Superalloys 1996 (1996), p. 191

[3] N. Zhou, C. Shen, M.J. Mills, Y. Wang: Acta Mater. Vol 56 (2008), p. 6156

[4] A. Gaubert, Y. Le Bouar, A. Finel : Phil. Mag.Vol 90 (2010), p. 375

[5] L. Méric, P. Poubanne, G. Cailletaud : J. Eng. Mater. Technol. Vol 113 (1991), p. 162

[6] D. Bettge, W. Österle: Scripta Mat. Vol 40 (1999), p. 389

[7] D. Ayrault : Fluage à haute température de superalliages base nickel monocristallins, $\mathrm{PhD}$ thesis (1989).

[8] H. Frenz, J. Kinder, H. Klingelhöffer, P.D. Portella: Superalloys 1996 (1996), p. 305 